\title{
Cervical Cord Compression With Lower Limbs Sensory Disturbance Presentation: Three Case Reports and Literature Review
}

\section{Yafei Cao}

Shenzhen Traditional Chinese Medicine Hospital

\section{Yihong Wu}

Shenzhen Traditional Chinese Medicine Hospital

\section{Weiji Yu}

Shenzhen Traditional Chinese Medicine Hospital

\section{Weidong Liu}

Shenzhen Traditional Chinese Medicine Hospital

\section{Shufen Sun}

Shenzhen Traditional Chinese Medicine Hospital

\section{Kun Gao ( $\nabla$ xiuqianyu@126.com )}

Shenzhen Traditional Chinese Medicine Hospital https://orcid.org/0000-0003-1400-286X

\section{Case report}

Keywords: Cervical spine, Cervical cord compressive myelopathy, False localizing, Tract pain

Posted Date: November 15th, 2021

DOI: https://doi.org/10.21203/rs.3.rs-1037842/v1

License: (c) (1) This work is licensed under a Creative Commons Attribution 4.0 International License. Read Full License 


\section{Abstract}

\section{Background:}

Lower limb sensory disturbance presentation can be a false localizing cervical cord compressive myelopathy (CSM). It may lead to delayed or missed diagnosis, resulting in the wrong management plan, especially in the presence of concurrent lumbar lesions.

\section{Case presentation:}

Three Asian patients with lower limb sensory disturbances presentation were treated ineffectively in the lumbar. Magnetic resonance imaging (MRI) showed cervical disc herniation and cervical level spinal cord compression. Anterior cervical discectomy surgery and zero-p interbody fusion were performed. After operations, imagings showed that the spinal cord compression were relieved, and the lower limbs sensory disturbances were also relieved. Three-months follow-up after operation showed good recovery.

\section{Conclusions:}

These three cervical cord compression cases of lower limb sensory disturbance presentation were easily misdiagnosed with lumbar spondylosis. Anterior cervical discectomy and fusion operation had a good therapeutic effect. Therefore, cases that present with lower limb sensory disturbance, but in a nonradicular classical pattern, should always alert a suspicion of a possible cord compression cause at a higher level.

\section{Introduction}

Cervical cord compressive myelopathy (CSM) is characterized by spinal cord compression or ischemia, followed by dysfunction due to the spinal canal sagittal diameter narrowing [1-2]. The typical symptoms of this disease are neck and shoulder pain, arm numbness or paresthesia, and gait disorders[3]. However, some patients have atypical symptoms, especially in the presentation of lower limbs, which difficult the diagnose $[4,5,6,7]$. These presentations are considered cervical cord compression "false localizing" since there are discrepancies between the neurological signs and the expected lesion anatomical locus [8].

We will present three rare cases of patients with lower limb sensory disturbance and cervical disc herniation, who were cured with anterior cervical surgery. Our study was approved by the Ethical Committee (EC) office of The Shenzhen Traditional Chinese Medicine Hospital and the subjects' rights were protected. Patients knew all rights and interests and signed the informed consent.

\section{Case Presentations}

\section{Case 1}




\section{Clinical presentation}

The first patient was an Asian male, 61, presenting left lower limb burning in the last 5 years. He also felt nack pain, upper limb numbness, and girdle chest sensation. There was no hypertension, diabetes, and nephropathy history. Also, he did not have a history of hepatitis, tuberculosis, trauma, blood transfusion, poisoning, and penicillin anaphylaxis.

On examination, he could walk on his own but staggering. The cervical spine motion range was normal, but the neck and shoulder muscles were tense. He also presented left body hypoesthesia below the nipple. The main left limb muscles strengths were slightly decreased, and the right ones were normal: (left biceps brachii $4 / 5$, triceps brachii $4 / 5$, dorsal forearm $4 / 5$, volar forearm $4 / 5$, hand grasp $4 / 5$ ); (right biceps brachii $5 / 5$, triceps brachii $5 / 5$, dorsal forearm $5 / 5$, volar forearm $5 / 5$, hand grasp $5 / 5$ ); (left quadriceps femoris $4 / 5$, tibialis anterior $4 / 5$, gastrocsoleus $4 / 5$, flexor longus $4 / 5$ ); (right quadriceps femoris $5 / 5$, tibialis anterior $5 / 5$, gastrocsoleus $5 / 5$, flexor longus $5 / 5)$; Bilateral Eaten signs $(-)$, Spurling signs (+), Hoffman signs (+), Babinski signs (+), patellar clonus (+++), ankle clonus (+++); Bilateral kneetendon reflex $(++)$ and Achilles tendon reflex $(++)$.

\section{Radiologic evaluation}

MRI and coronal and sagittal Computerized Tomography (CT) showed cervical disc herniation in cervical 3/4, 4/5, 5/6; and cervical 4/5; 5/6 level spinal cord compression (Fig. 1A-D).

\section{Treatment}

An anterior cervical surgery, C4/5, 5/6 discectomy, and zero-p interbody fusion were performed.

\section{Outcome and follow-up information}

After surgery, CT showed that the herniated discs in C4/5 and C5/6 were removed, and the fusion cage was in a good position (Fig. 2A-C). On the first day after the operation, the left lower limb burning was relieved, and upper limb numbness almost disappeared. One month later, he had no complaints about left lower limb burning and upper limb numbness in the phone call back.

\section{Case 2}

\section{Clinical presentation}

The second patient was an Asian female, 40, who came to our hospital due to left lower leg and foot numbness and pain in the last 6 months. She also felt headache, dizziness, right ring finger numbness, and right heel pain. She had diabetes for 6 years but in good control. She did not have a history of hypertension and nephropathy, no hepatitis, tuberculosis, trauma, blood transfusion, poisoning, and penicillin anaphylaxis. She did not regularly drink alcohol and never smoked. On examination, she could normally walk on her own. The cervical spine motion range was normal, but the neck and shoulder muscles were tense. She also had lumbar percussion pain. The left thigh circumference was about 1.5 
$\mathrm{cm}$ smaller than that of the opposite side. The left thigh sensation was decreased in the front and back sides, and the left leg and sole sensation were also decreased, especially the sole. The left limb main muscles strength was slightly decreased. Additionally, the right heel had tenderness. The pathological and physiological reflexes were normal.

\section{Radiologic evaluation}

MRI showed cervical disc herniation (cervical 4/5,5/6) and cervical 5/6 level left spinal cord compression (Fig. 3A-C). Cervical 5 had vertebral hemangioma and left ligamentum flavum thickening at the T2/3 disc level.

\section{Treatment}

An anterior cervical surgery, C4/5, 5/6 discectomy, zero-p interbody fusion, and a C5 cement filling was performed.

\section{Outcome and follow-up information}

After surgery, the CT showed that C4/5, 5/6 spinal cord compression was relieved (Fig. 4A-C). On the first day after the operation, the left lower leg and foot numbness and pain were relieved, the right heel pain disappeared. The left limb's main muscles strength was recovered. The headache, dizziness, right ring finger numbness were slightly relieved. One month later, she had no complaints about lower limb numbness and pain in the phone call back.

\section{Case 3}

\section{Clinical presentation}

The third patient was an Asian male, 34, who came to our hospital due to right lower limb numbness and pain in the last 3 months. He had no history of hypertension, diabetes, and nephropathy; no hepatitis, tuberculosis, trauma, blood transfusion, poisoning, and penicillin anaphylaxis. He did not drink alcohol regularly and never smoked.

On examination, he could normally walk on his own. The right body hypoesthesia below the nipple and right lower leg temperature sensation declined. The limbs muscle tension was normal, and there was no obvious atrophy and fine motor disorder. The cervical spine motion range was normal, but the cervical spine curvature was straight, and the neck and shoulder muscles were tense. The main limbs muscles' strength was normal. The pathological and physiological reflexes were also normal.

\section{Radiologic evaluation}

Magnetic resonance imaging (MRI) showed cervical disc herniation (cervical 5/6); cervical 5/6 level left spinal cord compression and degeneration (Fig. 5B, C). The whole spine MRI showed no thoracic and lumbar abnormalities (Fig. 5A).

\section{Treatment}


An anterior cervical C5/6 surgery, a discectomy, and a zero-p interbody fusion were performed.

\section{Outcome and follow-up information}

After surgery, the MRI showed that the left spinal cord C5/6 compression was relieved (Fig. 6A, B). After the operation, right body numbness and pain below the nipple cleared up and the right lower limb numbness and the pain was relieved. Three months later, the MRI showed that the spinal cord degeneration had recovered, and the right lower limb numbness and pain were almost relieved.

\section{Discussion And Conclusions}

In the three reported patients, symptoms did not appear typically as neck pain and upper limbs numbness, instead, they had lower limbs sensory disturbance presentation. This presentation is considered cervical cord compression "false localizing" since there are discrepancies between neurological signs and the expected lesion anatomical locus [8]. James Collier [9] found discrepancies between antemortem clinical features with expected postmortem anatomical findings and it was firstly named "False localizing signs" in 1904. The correct diagnosis was markedly delayed because of the false localizing.

In case 3 , the patient suffered from right lower limb numbness and pain in the last 3 months. He was young and visited many orthopedic doctors. Most doctors believed that it was caused by lumbar vertebrae, although the MRI did not show lumbar abnormalities, and conservative lumbar treatments were ineffective. The right body below the nipple alerted us to the possibility of cervical cord involvement. The case 1 patient presented left lower limb burning in the last 5 years nack pain, upper limb numbness, and chest girdle sensation. Although his main symptoms were not located in the cervical spine, combined with secondary symptoms we realized that the cervical cord compression would be the main cause. The case 2 patient came to the hospital due to left lower leg and foot numbness and pain and felt headache, dizziness, right ring finger numbness, and right heel pain. Her symptoms were complicated and accompanied by lumbar disc herniation showed by MRI. These three cases have similarities: lower limbs sensory disturbance; cervical cord compression; and the symptoms improved after cervical spine surgery. Case 2 and case 3 presented a chest girdle sensation that disappeared after surgery. However, case 1 had no girdle sensation but presented right heel pain that we missed as a calcaneal spur. Imedeadlty after surgery, the right heel pain disappeared.

The precise lower limb sensory disturbance mechanism caused by cervical cord compression is not clearly understood. The pain in the lower body parts caused by different spinal cord pathological conditions is called "tract pain," and the precise mechanism responsible for such pain is unclear. In most reported cases, the tract pain was the main symptom, and usually described as burning, boring, or aching [10]. The spinal tracts disorder was regarded as the main reason for tract pain in previous reports [1112]. From the outside to the inside, the spinothalamic tract fibers were sacral, lumbar, thoracic, and cervical. The cervical segment fibers were in most medial and scattered positions. The compression lesion finally affected the cervical segment fibers and the segments lower than the lesion plane. The 
spinal cord blood supply should also be considered, especially in patients with girdle sensation. The spinal cord T4 level sorroundings is a watershed zone of the anterior spinal artery (ASA). The thoracic cord around the T4 level will become ischemic if the ASA is compressed by a lower cervical lesion [13]. Additionally, interruption of pain modulation pathways was considered as an important reason for this tract pain. In the presumed pathomechanism, descending antinociceptive projections interruptions from the rostral ventral medulla (RVM) play a critical role in the modulation and maintenance of the pain threshold [14].

The above pathological mechanisms were speculated, but the only confirmed point is that cervical surgery can eliminate the lower limb's sensory disturbance. The disease diagnosis is very important, especially for those who have concurrent lumbar spinal lesions, and it is often confused as a lumbar problem, leading to lumbar treatment or even unnecessary lumbar surgery. How to avoid this misdiagnosis? Based on careful physical examination, a more comprehensive inspection should be performed. Although the main symptoms are in the lower limbs, CSM should be considered when the accompanying symptoms appear above the lumbar level. Case 1 and case 3 presented girdle sensation, and case 2 presented headache, dizziness, right ring finger numbness. Additionally, for those patients with both lumbar and cervical lesions on MRI, cervical epidural steroid block, given either in a foraminal or interlaminar approach, would be helpful to differentiate compressive cervical and lumbar stenotic lesions [6].

Therefore, we demonstrated three cervical cord compression cases presenting lower limb sensory disturbance. These cases were probably caused by spinothalamic tract disorder, descending antinociceptive projections interruptions from RVM, or compressed ASA. They were easily misdiagnosed as lumbar spondylosis. Anterior cervical discectomy and fusion operation had a good therapeutic effect. Thus, cases presenting with lower limb sensory disturbance, but in a non-radicular classical pattern, should always alert a suspicion to a possible cord compression cause at a higher level. These case studies will help to better deal with CSM presenting lower limb sensory disturbance.

\section{Abbreviations}

cervical cord compressive myelopathy (CSM); coronal and sagittal computerized tomography (CT); magnetic resonance imaging (MRI); Ethical Committee (EC); anterior spinal artery (ASA); rostral ventral medulla (RVM).

\section{Declarations}

\section{Acknowledgements}

NONE

\section{Funding}


Sanming Project of Medicine in Shenzhen ( NO.SZSM201812066 )

\section{Contributions}

Yafei Cao and Gao Kun planned and designed the case report and literature review. Weiji Yu, Weidong Liu,and Shufen Sun collected and reviewed the literature on the subject and drafted the paper. Yihong Wu and Gao Kun performed the draft revision and collected clinical and pathological data. All authors read and approved the final manuscript

\section{Ethics declarations}

\section{Ethics approval and consent to participate}

Our work was approved by the Ethics Committee of Shenzhen Traditional Chinese Medicine Hospital, and written informed consent was obtained from the patient.

\section{Consent for publication}

Written consent for publication of this case and clinical dates was informed and obtained from the patient.

\section{Competing interests}

All authors have declared that no conflict of any interests.

\section{Availability of data and material}

The datasets used and/or analysed during the current study available from the corresponding author on reasonable request.

\section{References}

1. Gorter K. Influence of laminectomy on the course of cervical myelopathy. Acta Neurochir (Wien) 1976;33(3-4):265-281

2. Gross J, Benzel E. Dorsal surgical approach for cervical spondylotic myelopathy. In: Camins MD, ed. Techniques in Neurosurgery. Philadelphia: Lippincott Williams \& Wilkins; 1999:162-176

3. Montgomery DM, Brower RS. Cervical spondylotic myelopathy. Clinical syndrome and natural history. Orthop Clin North Am. 1992; 23:487.

4. Ross MD, Elliott R. Cervical cord compressive myelopathy in a man with a primary complaint of knee pain. J Spinal Cord Med. 2010;33(4):431-434.

5. Akhavan-Sigari R, Rohde V, Alaid A. Cervical spinal canal stenosis and central disc herniation c3/4 in a man with primary complaint of thigh pain. J Neurol Surg Rep. 2013;74(2):101-104. 
6. Chan CK, Lee HY, Choi WC, Cho JY, Lee SH. Cervical cord compression presenting with sciatica-like leg pain. Eur Spine J. 2011;20:S217-21

7. Kozaki T, Minamide A, Iwasaki H, Yuakawa Y, Ando M, Yamada H. Funicular pain: a case report of intermittent claudication induced by cervical cord compression. BMC Musculoskelet Disord. 2020;21(1):302.

8. Kobayashi S. "Tract pain syndrome" associated with chronic cervical disc herniation. Hawaii Med J. 1974;33(10):376-381.

9. Collier J. The false localizing signs of intracranial tumour.Brain. 1904; 27:490-508

10. Neo M, Ido K, Sakamoto T, Matsushita M, Nakamura T. Cervical disc herniation causing localized ipsilateral popliteal pain. J Orthop Sci. 2002;7(1):147-150.

11. Scott M. Lower extremity pain simulating sciatica: tumors of the high thoracic and cervical cord as causes. JAMA.1956; 160:528-534

12. Pego-Reigosa R, Trobajo de las Matas JE, Branas F, Martinez-Vazquez F, Cortes-Laino JA. Dorsal sensory level as a false localizing sign in cervical myelopathy [in Spanish]. Rev Neurol 1998;27:86-8.

13. Ochiai H, Yamakawa Y, Minato S, Nakahara K, Nakano S, Wakisaka S. Clinical features of the localized girdle sensation of mid-trunk (false localizing sign) appeared in cervical compressive myelopathy patients. J Neurol 2002;249:549-53.

14. Thimineur M, Kitaj M, Kravitz E, Kalizewski T, Sood P .Functional abnormalities of the cervical cord and lower medulla and their effect on pain: observations in chronic pain patients with incidental mild Chiari I malformation and moderate to severe cervical cord compression. Clin J Pain .2002;18:171

\section{Figures}



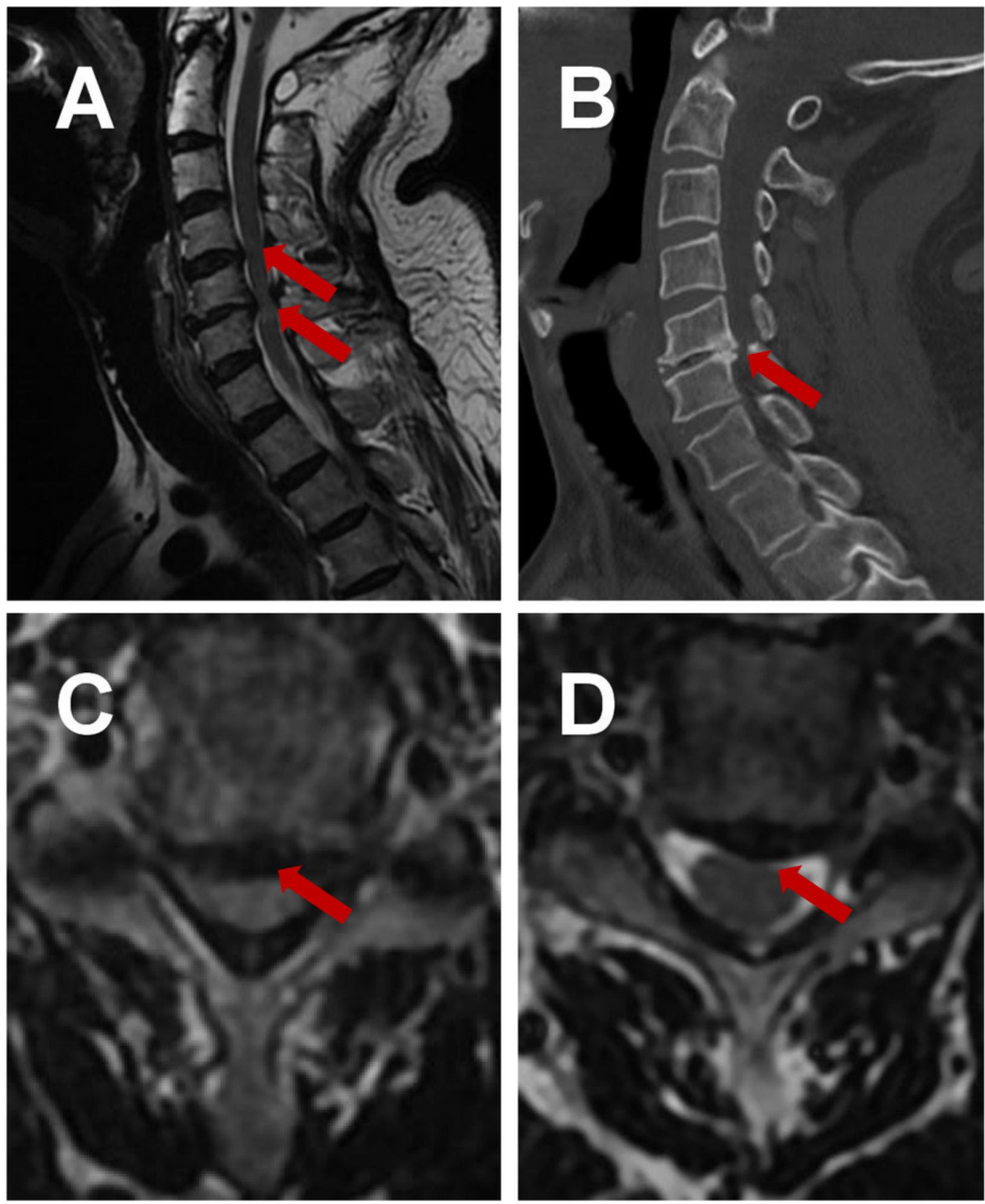

\section{Figure 1}

Preoperative imaging of the cervical spine: (A) Axial and (C) sagittal MRI showed C5/ 6 level spinal cord compression; (B) Axial and (D) sagittal CT showed intervertebral space narrowing, osteophyte formation, and osteosclerosis. 

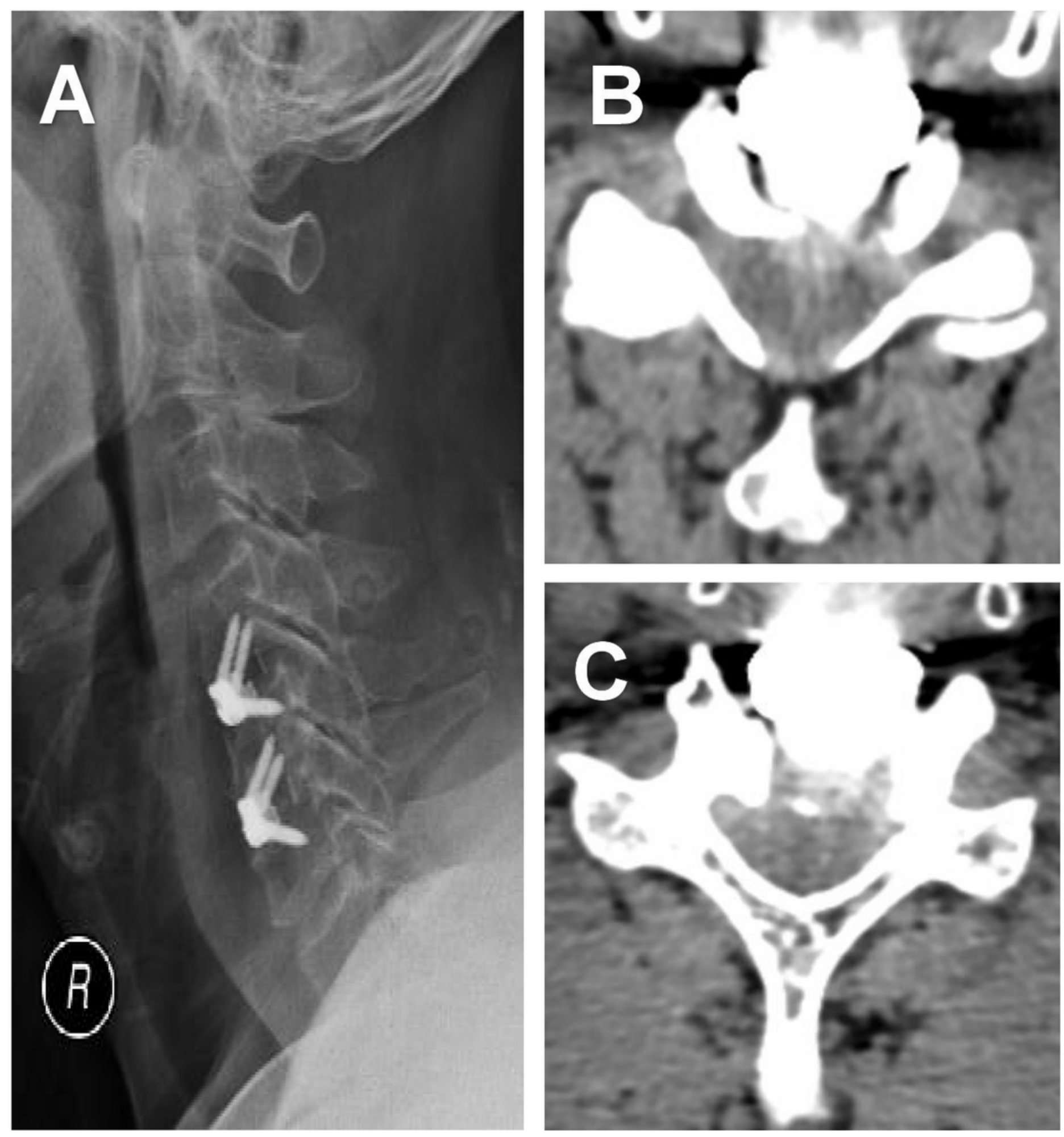

\section{Figure 2}

Postoperative imaging of the cervical spine: (A) X-Ray of the C4/5, C5/6 anterior cervical surgery, discectomy, and zero-p interbody fusion; (B) Axial and (C) sagittal CT showed intervertebral space narrowing, osteophyte formation, and osteosclerosis. 

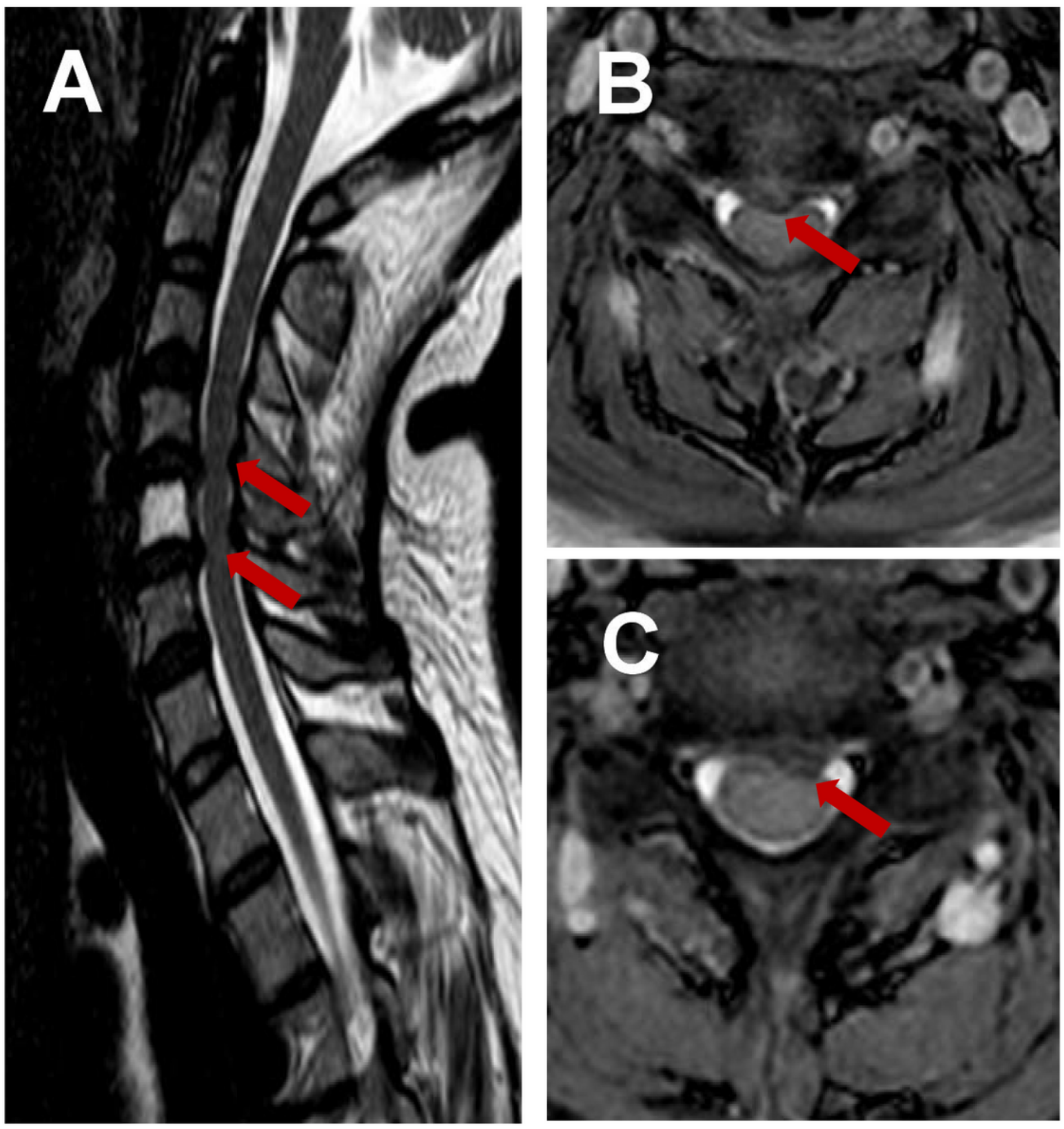

\section{Figure 3}

Preoperative imaging of the cervical spine: $(A)$ Axial and (B, C) sagittal MRI showed C4/5, C5/ 6 level spinal cord compression. 

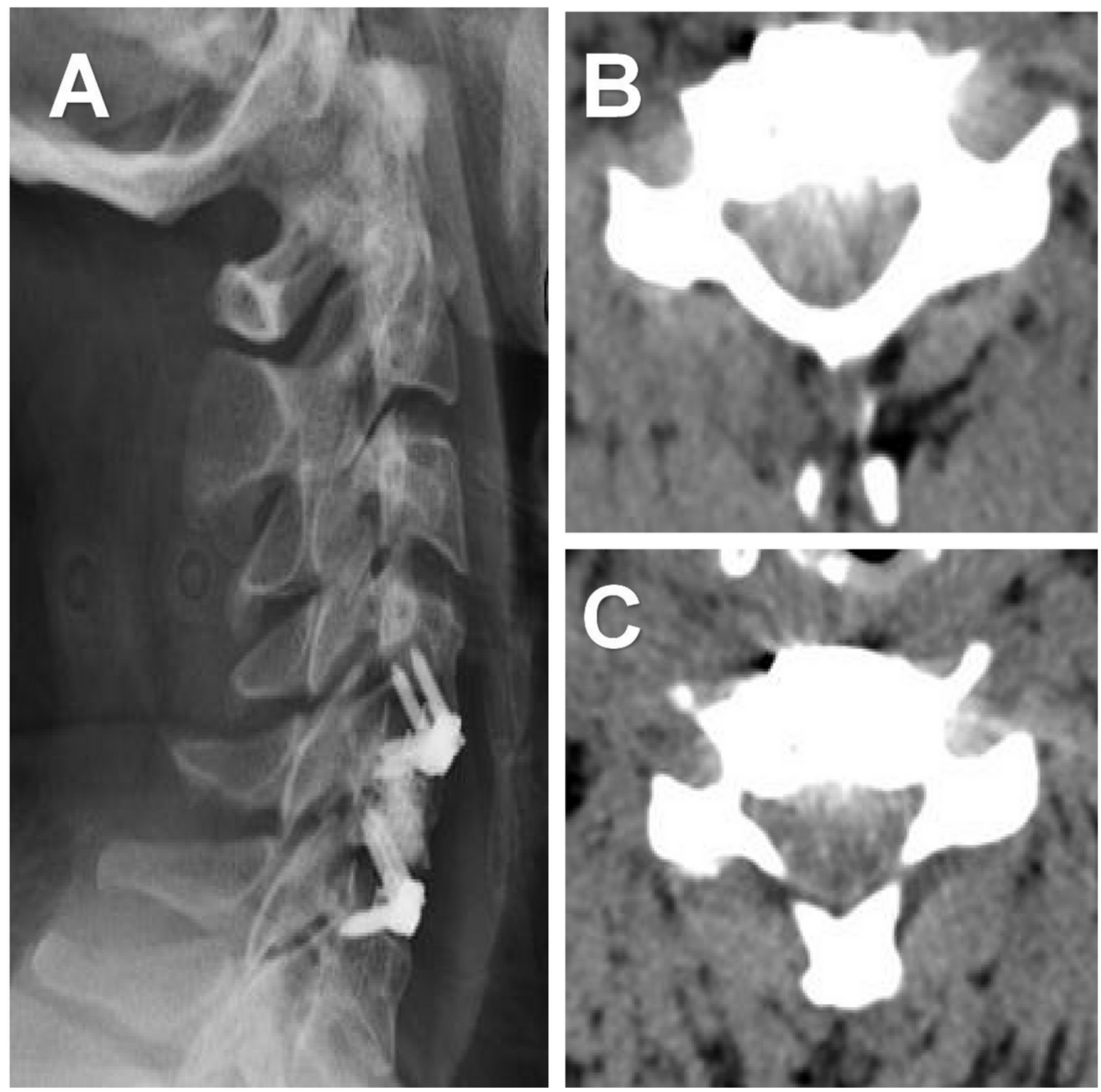

Figure 4

Postoperative imaging of the cervical spine: (A) X-Ray of the C4/5, C5/6 anterior cervical surgery, discectomy, and zero-p interbody fusion; (B, C) Sagittal CT showed C4/5, C5/6 spinal cord compression relieved after surgery. 


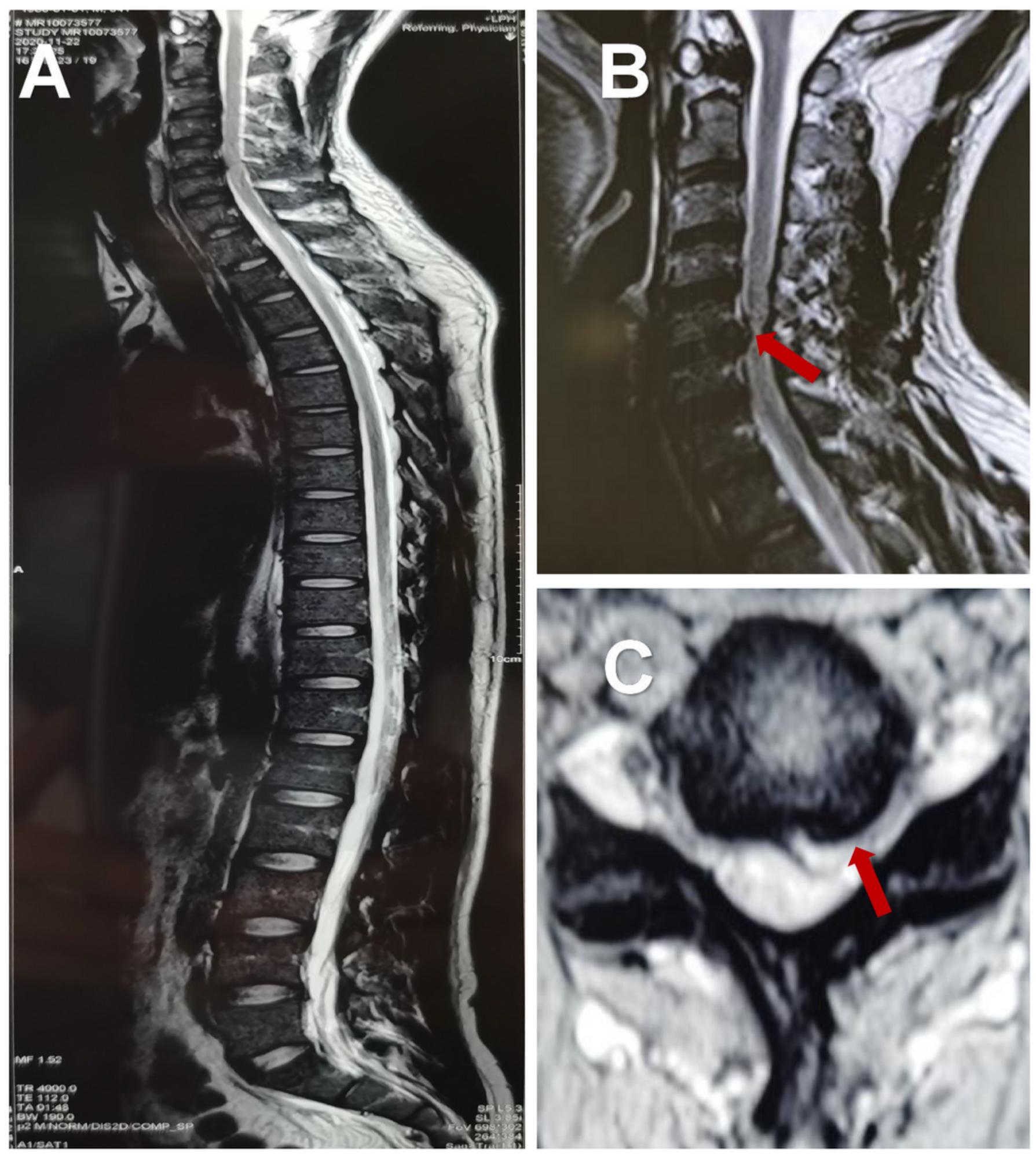

Figure 5

Preoperative and postoperative cervical spine imaging: (A) Whole spine MRI shows normal lumbar and thoracic; (B) Axial and (C) sagittal MRI showed C5/6 level left spinal cord compression; 


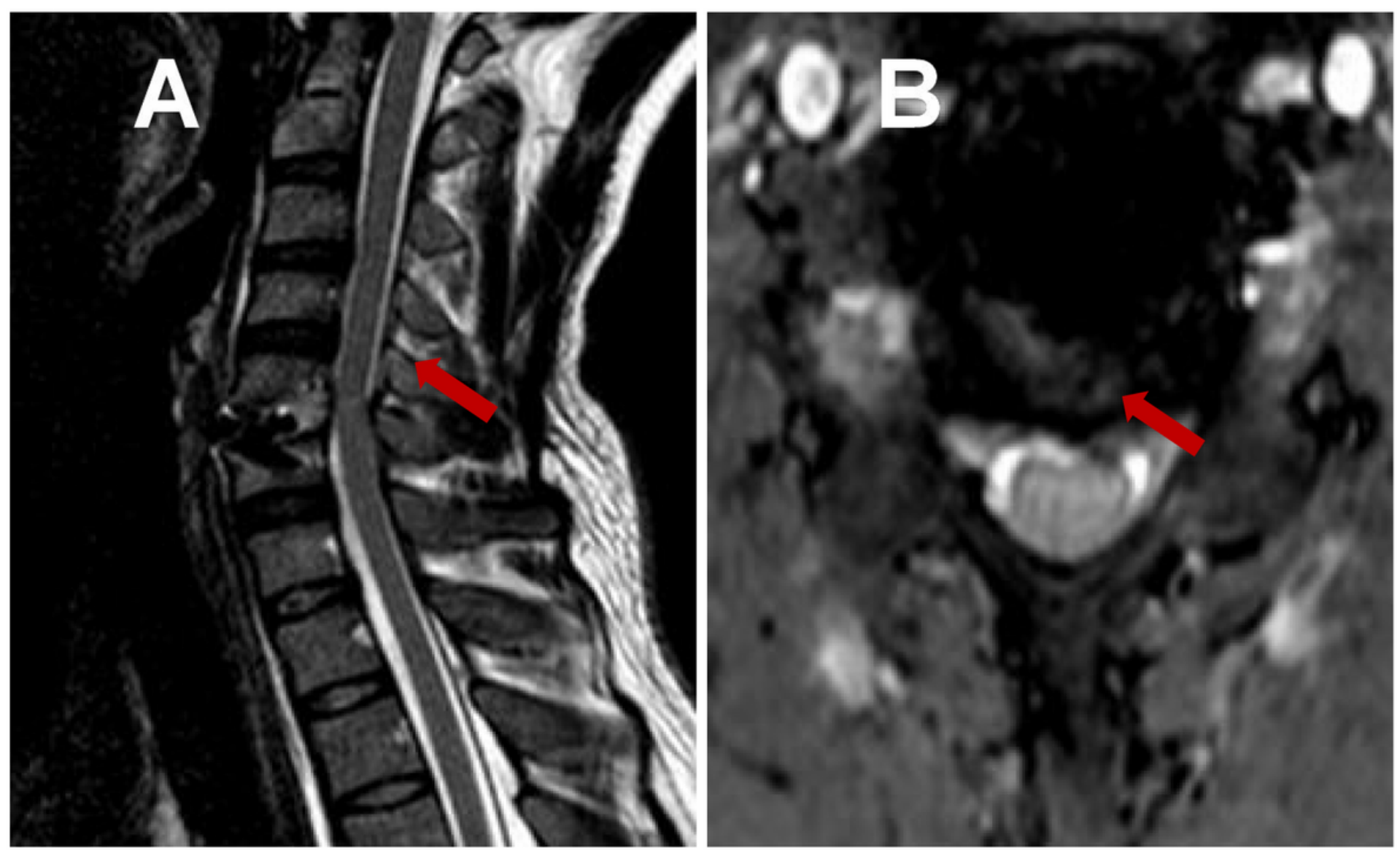

Figure 6

Postoperative cervical spine imaging: (A) Axial and (B) sagittal MRI showed C5/6 left spinal cord compression relieved after surgery.

\section{Supplementary Files}

This is a list of supplementary files associated with this preprint. Click to download.

- CAREchecklistEnglish2013.pdf 